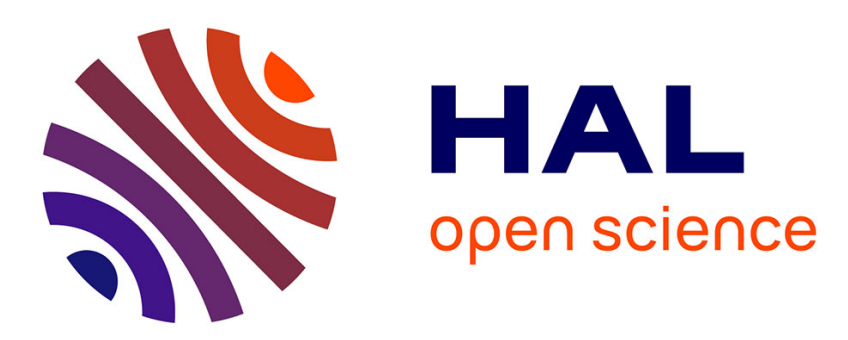

\title{
Facebook à l'épreuve de la différence. Avatars du narcissisme des petites différences \\ Rémy Potier
}

\section{To cite this version:}

Rémy Potier. Facebook à l'épreuve de la différence. Avatars du narcissisme des petites différences. Topique - Revue freudienne, 2012, Le narcissisme des petites différences, 4 (121), pp.97 - 109. 10.3917/top.121.0097 . hal-01516377

\section{HAL Id: hal-01516377 \\ https://hal.science/hal-01516377}

Submitted on 24 Jul 2017

HAL is a multi-disciplinary open access archive for the deposit and dissemination of scientific research documents, whether they are published or not. The documents may come from teaching and research institutions in France or abroad, or from public or private research centers.
L'archive ouverte pluridisciplinaire HAL, est destinée au dépôt et à la diffusion de documents scientifiques de niveau recherche, publiés ou non, émanant des établissements d'enseignement et de recherche français ou étrangers, des laboratoires publics ou privés. 


\title{
Facebook à l'épreuve de la différence Avatars du narcissisme des petites différences
}

\author{
Rémy POTIER
}

L'usage des réseaux sociaux tels que Facebook est aujourd'hui très répandu. Cette pratique sociale fait partie intégrante du quotidien des jeunes générations. C'est un fait social qui témoigne de l'intégration d'une nouvelle technologie de la communication dans la vie des sujets contemporains, cette pratique s'installe après les blogs ${ }^{1}$ comme modalité d'expression de soi et d'interaction avec les autres. Plus de 25 millions d'utilisateurs sont recensés aujourd'hui ${ }^{2}$, c'est dire l'importance du phénomène et donc l'intérêt d'une élucidation des codes, des usages comme des modalités d'interactions qui seuls permettront d'articuler ce que les patients en rapportent, et ainsi permettre de mieux entendre ce qui y est engagé sur le plan psychique.

Facebook permet de communiquer avec d'autres personnes, en créant des liens d'amitiés et de partage d'informations, comme des images, des vidéos, des articles de presse, etc. Plusieurs façons d'échanger sont ainsi possibles; à partir d'une même page personnelle, des mails s'échangent, des discussions instantanées se déroulent constamment, les photos et les vidéos se partagent ainsi que des articles divers, enfin des commentaires et des appréciations nouent les liens entre les réseaux d'amis. La place que prend ce média dans la société, le fait d'en entendre de plus en plus souvent parler par les patients en lieu et place de leur relation, encourage à y repérer les spécificités pour y retrouver les modalités d'expressions psychiques, bien différentes selon la structure du sujet. Les réseaux sociaux sont bien investis comme espace d'échange et d'interaction mais dans une intimité particulière selon le rapport entretenu avec les objets techniques.

1. Potier, R., \& Bialès, P. (2007). de l'amitié en virtuel. Adolescence, 61(3), 581.

2. http://www.socialbakers.com/Facebook-statistics/france 
André Leroi Ghouran a montré comment les outils techniques participent du processus d'hominisation ${ }^{3}$. Chaque époque métabolise son progrès, change l'homme en le rendant dépendant des techniques qui lui ouvrent des possibles. Cette donnée anthropologique est confirmée par le psychanalyste Harold Searle dans L'environnement non humain. Selon Searle, l'élément non humain de l'environnement de l'homme forme l'un des constituants les plus fondamentaux de la vie psychique, la capacité ou l'incapacité de l'humain à entretenir avec son entourage non humain une relation constructive, contribue à son équilibre ou son déséquilibre psychique.

Il me paraît intéressant de se saisir du concept de narcissisme des petites différences pour le revisiter à l'heure où la dimension virtuelle de l'image a une place prépondérante dans les pratiques sociales. L'expérience consacrée par les réseaux sociaux confine le sujet dans un rapport à l'altérité tout à fait singulier et nouveau, portfolio de collections d'amis, venant signifier une popularité, une identité numérique. des spécificités intéressantes peuvent être mises en avant pour dégager les enjeux cliniques sur le plan individuel et collectif.

\section{LA MÉTAPHORE VIRALE AU CEUR dES RÉSEAUx SOCIAUx}

Le virtuel fait partie intégrante de notre environnement, notre façon d'y interagir dépend de la façon dont nous parvenons à métaboliser ces nouvelles technologies, le normal et le pathologique s'y côtoient donc nécessairement. Mon propos à l'égard des réseaux sociaux peut ici s'inscrire dans la suite du travail de Sylvain Missonnier qui a proposé le terme de «psycho(patho)logie du virtuel quotidien $»^{4}$.

dans cet esprit, les pratiques dont témoignent les patients lorsqu'ils évoquent Facebook sont un indice précieux de ce qui se questionne en eux-mêmes depuis leur narcissisme et l'épreuve que constitue pour eux la relation à autrui. Pour les adolescents, il s'agit souvent d'un mode d'approche rassurant et feutré, une mise à l'abri provisoire, un pansement peut-être au moment du «pubertaire ${ }^{5}$. Nous disposons aujourd'hui de nombreuses études intéressantes sur les réseaux sociaux, en sociologie, en économie, en sciences politiques voire dans d'autres domaines de la psychologie ${ }^{6}$. dans un langage conceptuel différent des intérêts propres à la psychanalyse, ces études donnent l'occasion d'une interaction féconde pour articuler la clinique individuelle aux effets analysables sur le plan

3. Leroi Ghouran, A., Le Geste et la Parole, 1. : Technique et langage, 2. : La Mémoire et les Rythmes, Paris, Albin Michel, 1964-1965.

4. Missonnier, S., et Lisandre, H., (sous la direction de), (2003), Le virtuel-la présence de l'absent, Paris, EdK.

5. Philippe Gutton, Le pubertaire, (2003), Le pubertaire. PUF.

6. Voir bibliographie et suite de l'article. 
collectif. Ces études permettent de nous aider à répondre aux questions suivantes: Quel type d'usages sont rendus possibles par Facebook ? de quelles expériences parle-t-on? En quoi le narcissisme des petites différences offre-t-il l'occasion d'une élucidation de certaines problématiques rencontrées dans la clinique et ouvre vers des questions concernant le malaise actuel dans la culture (Richard, F., 2012) ? ${ }^{7}$

Les réseaux sociaux offrent donc un espace d'échange dont il résulte des modalités d'interactions particulières. dans un article récent ${ }^{8}$ Jérôme Batout (2011) épingle 1'un des traits particuliers de Facebook comme espace social, l'aconflictualité. Facebook proposerait une «utopie sociale» (Batout, 2011). La dimension sociale ici en jeu se joue dans le refoulement de toute conflictualité apparente. Il existe cependant des occasions de disputes sur certaines pages, rarement entre amis mais entre «fans » ou contradicteurs de certaines causes. Si ce conflit-là s'y trouve exprimé, c'est de façon fugace et réactive, trace d'une interaction immédiate qui laisse vite place à l'annulation de l'autre et à sa suppression dans l'espace visible des échanges. On peut bloquer l'inopportun, paramétrer son compte pour se garantir l'expérience aconflictuelle offerte par les efforts de rationalisation du groupe Facebook. La belle indifférence y est au fond l'expérience la mieux partagée. Le problème de cette aconflictualité pour la psychanalyse apparaît dès lors évident dans la mesure où la clinique nous enseigne combien le conflit est structurant pour la personnalité. La socialité construite sur Facebook prend bien il est vrai le risque d'un vide : les liens s'y tissent selon les affinités exclusives, à l'exclusion des différences concrètes dont par ailleurs l'ensemble du réseau et de ses échanges témoignent profondément. Or c'est sans doute dans ce vide que le narcissisme des petites différences se ressources s'y déploie dans de multiples symptômes.

Facebook est donc le site d'une génération, site sur lequel les dimensions conflictuelles et contradictoires sont refoulées, évitées voire déniées.

Suite à cette présentation du réseau social, je souhaite attirer l'attention sur l'un de ses enjeux pour bon nombre d'utilisateurs, l'extension de sa « viralité» ${ }^{9}$. Est «viral» dans le vocabulaire lié à internet, ce qui se propage et témoigne de son extension. Empruntée à l'expérimentation des virus informatiques, cette métaphore offre l'idée d'une stratégie d'extension du domaine de sa popularité online. Il est vrai que l'enjeu recherché par les informaticiens réalisant les virus informatiques à l'encontre de la firme Microsoft, était d'acquérir à travers la nuisance réalisée par leur virus, une popularité imaginaire incontestée, constatée par les victimes et reconnue par les pairs. La dimension narcissique était déjà en

7. Richard, F., (2011), Les formes actuelles du malaise dans la culture. Recherches en Psychanalyse, 11.

8. Batout, J., (2011), Le monde selon Facebook. Le Débat, 163.

9. Les Américains usent de cette métaphore de façon à exprimer la propagation d'une information, d'une idée ou tout simplement de quelque chose : it goes viral. 
place. Les hackers en recherche d'emploi ont pu à maintes reprises s'illustrer dans l'atteinte d'un système de sécurité d'une entreprise afin de démontrer à leur employeur potentiel la nécessité de les embauchés : véroler un système étant le meilleur moyen de se positionner comme étant le meilleur pour éviter une telle intrusion. Est aujourd'hui viral ce qui fait le buzz, celui qui peut se targuer d'un nombre important d'amis, de «fans », ou encore de «followers » dans le vocabulaire issu de Twitter.

La métaphore virale peut être interrogée à l'épreuve du concept de narcissisme des petites différences. Au menu des agences de marketing, la viralité est offerte aux entreprises et aux usagers comme une stratégie valorisée et nécessaire pour «exister ${ }^{\mathbf{1 0}}$. Cet usage peut être mis en lumière par les patients qui témoignent précisément d'une préoccupation concernant la viralité de leur identité virtuelle.

\section{L'IdENTIFICATION VIRALE COMME RÉVÉLATEUR dU NARCIS- SISME dES PETITES dIFFÉRENCES}

L'identification est un processus psychique inconscient par lequel une personne rend une partie plus ou moins importante de sa personnalité conforme à celle d'un autre qui sert de modèle. Mode premier de la relation aux autres, son assomption est d'abord narcissique. Mais comment ce mécanisme constitutif de la vie psychique rencontre-t-il le virtuel ? Que se joue-t-il potentiellement au sein des réseaux sociaux à cet égard ?

Il apparaît intéressant de revisiter l'intrication de ces concepts et de s'attarder sur la notion de viralité, telle qu'elle est utilisée, tant du point de vue individuel que collectif. Pour ce faire, il convient de confronter notre méthodologie de recherche psychanalytique aux résultats issus de différentes études consacrées à ce thème. Ces études sont intéressantes dans la mesure où elles nous aident à discriminer la part du normal et du pathologique (au sens de Canguilhem) voire de repérer la dimension pathologique d'une certaine conception normative résultant de ces usages du virtuel quotidien, cette seconde dimension faisant signe vers l'actuel malaise dans la culture, dont le souci réifié de l'expression de soi se heurte à un effet de standardisation.

Je me limiterai dans le cadre de cet article à quelques études récentes concernant les usages de Facebook.

Gonzales et Hancock (2011) ${ }^{\mathbf{1 1}}$ ont étudié l'effet de l'utilisation de Facebook sur l'estime de soi. Les résultats de leur étude révèlent que prendre conscience de soi, en regardant son propre profil sur Facebook, en améliore l'estime.

10. http://www.abc-netmarketing.com/-Marketing-viral-.html

11. Gonzales, AL, Hancock, JT., Mirror, mirror on my Facebook wall : effects of exposure to Facebook on self-esteem. Cyberpsychol Behav Soc Netw $2011 ; 14: 1-2$. 
L'article indique que choisir la manière dont on se présente sur les médias numériques conduit à accroître son potentiel relationnel et influence également l'aperception. Ainsi selon ces auteurs, la page Facebook investie fonctionne comme soutien, étayage à l'estime de soi. Il faudrait pourtant distinguer l'investissement de la mise à jour de son profil avec la recherche spécifique de viralité, terme devenu marketing, qui correspond à une activité spécifique et qui peut être davantage assimilable à l'hystérie en tant que telle. Pour autant, cette estime de soi mériterait d'être éclairée davantage que ne le propose concrètement cette étude. Le temps concédé à cette contemplation, son effet de standardisation et d'assimilation ne sont pas interrogés. Le fait également que le réseau d'amis fonctionne comme les témoins actifs - à travers leurs approbations - $m$ 'apparaît comme un indicateur d'un narcissisme des petites différences, fondé sur l'aconflictualité qui n'est autre qu'une réelle indifférence.

En France, Moreau, A., Roustit, O., Chauchard, E., \& Chabrol, H. (2012) ${ }^{\mathbf{1 2}}$ insistent sur le fait que Facebook est un espace virtuel qui a la particularité d'être nominatif et de regrouper les pairs et la famille. Ce trait apparaît positivement aux auteurs. Ce réseau social donne un accès permanent aux autres, ou au moins à des informations les concernant. Facebook permettrait à l'adolescent d'expérimenter les relations sociales et d'y jouer des conflits de séparation comme de dépendance. La clinique, comme nous le verrons, corrobore ce dénouement processuel, dans la mesure où la dimension narcissique de cette expérience s'équilibre d'un travail d'élaboration, notamment chez les adolescents limites au narcissisme fragile. En effet, si l'on veut bien suivre le fil du narcissisme des petites différences, à l'occasion d'une réflexion sur les réseaux sociaux, alors, il faut questionner davantage l'expérience de plaisir qui y est associé. Il faut ajouter l'idée que ces expérimentations confinent les usagers à une standardisation des pratiques qui offre peu de prise à l'expérience de l'autre et à sa différence. La chaleur aconflictuelle offerte par ce réseau social ne concerne d'ailleurs pas que les adolescents; elle rabat la notion de partage à l'expression de soi sous le regard des autres, ce qui n'est pas sans conséquence anthropologique.

Antoci, A., Sabatini, F., \& Sodini, M. (2012) ${ }^{\mathbf{1 3}}$, mettent quant à eux en avant trois raisons qui permettent de penser que Facebook fonctionne comme une stratégie individuelle pour contrer la pression du temps dans nos sociétés contemporaines $^{\mathbf{1 4}}$ : il permet le fait de se protéger de l'environnement social,

12. Moreau, A., Roustit, O., Chauchard, E., \& Chabrol, H., (2012), L'usage de Facebook et les enjeux de l'adolescence : une étude qualitative. Neuropsychiatrie de l'enfance et de l'adolescence, 60 (6), 429-434.

13. Antoci, A., Sabatini, F., \& Sodini, M., (2012), See you on Facebook ! A framework for analyzing the role of computer-mediated interaction in the evolution of social capital. The Journal of Socio-Economics, 41(5), 541-547. 
notamment en permettant la connexion avec des amis ; le fait également de permettre des interactions plus rapides que ce qui est permis en face à face, que ce soit durant les pauses au travail, qu'en attendant le train, etc.; enfin selon les auteurs, c'est le capital social online qui est privilégié. Cet article explicite les stratégies individuelles des usagers pour satisfaire à ces nouvelles données anthropologiques, ce qui fait signe pour cet article au malaise propre à notre époque et au rôle de Pharmakon ${ }^{15}$ que peuvent assumer les réseaux sociaux.

Enfin, dominique Piotet, $(2011)^{\mathbf{1 6}}$, dans la revue Esprit, fait remarquer que Facebook est devenu la plate-forme sociale incontournable sur laquelle jeunes et moins jeunes se retrouvent, et entretiennent des conversations. Mais ce site est devenu, viralité oblige, un point d'attraction et de présence obligatoire pour les entreprises, les partis politiques. Or, et c'est un des traits qui confine au narcissisme des petites différences, la plateforme qui connaît bien ses utilisateurs pour en analyser constamment les publications, offre aux usagers l'accès au contenu qui est précisément en lien avec ses intérêts propres. L'auteur offre une précision intéressante et trop peu mise en avant. Si le réseau social représente les personnes que l'on connaît, le «graphe social» ajoute les modalités de notre connexion aux autres : localisation, travail, centres d'intérêt... C'est précisément ce qu'est devenu Facebook. Ainsi, si mon réseau social est ma liste d'amis, le graphe social montre un peu plus que cela et assoit davantage la portée narcissique et stratégique de ce qu'il convient de montrer et de regarder chez l'autre...Faire partie du graphe social et participer à la conversation, où que nous nous trouvions, a nécessairement comme le dit l'auteur, un impact sur notre identité : «La façon dont nous interagissons, dont nous intervenons dans la société, avec nos amis, notre famille, mais aussi sur nous-mêmes, nos sentiments et la manière dont nous les partageons. Bref, se dessine un moi social dont la surface est désormais digitale. ${ }^{\mathbf{1 7}}$

de ces études concernant les usages qui caractérisent les réseaux sociaux, nous pouvons relever plusieurs éléments qui peuvent témoigner des enjeux narcissiques qui semblent déplacer le site du narcissisme des petites différences

14. "First, it is less exposed to the deterioration of the social environment that physically surrounds individuals. Online networking allows people to interact with friends and acquaintances even in distant locations. Second, it is less time consuming than face-to-face inter- action. So, it suffers less from the reduction in the time available for social participation. Social networking sites like Facebook and Twitter allow users to stay in touch with friends in their spare time, while sitting at a desk during their working day or while waiting for the train. Third, online interactions contribute to the accumulation of an "Internet social capital" which benefits solely Internet user", p. 542.

15. Potier, R., (2009), Au risque du virtuel, Topique 2/2009 ( $\left.{ }^{\circ} 107\right)$, p. 149-162. Suivant la proposition de J. derrida dans son célèbre commentaire du Phèdre de Platon : «,La pharmacie de Platon ,» (1968).

16. Piotet, d .,(2011), Comment les réseaux sociaux changent notre vie, Esprit, Juillet (7), p. 82 .

17. idem., p. 89. 
au sein d'une nouvelle « nation », Facebook :

- les attitudes narcissiques;

- les stéréotypes identitaires;

- le nombre d'amis, comme souci constant;

- les publications quotidiennes;

- l'importance accordée au regard de l'autre dans la représentation de soi ;

- l'indifférence à la différence ;

- la construction de l'image de soi ;

- les constellations d'intérêts auto-centrés.

dans la clinique, ces éléments font échos au récit de certains patients, précisément préoccupés de leur popularité ou de leur image véhiculée à partir de leur compte.

deux récits d'adolescents que je suis depuis plusieurs années me paraissent révélateurs des mécanismes psychiques induits et actualisés par l'importance des réseaux sociaux. Ces récits peuvent trouver à se répondre dans le fait qu'ils sont énoncés depuis une posture psychique différente, l'un témoignant d'une structure hystérique, l'autre d'une position obsessionnelle, les deux relevant la dimension narcissique propre à éclairer le concept de narcissisme des petites différences dans ce contexte. Corrélativement, il permet d'en interroger l'actualité au sein de notre civilisation.

Le premier récit résulte des associations et de l'élaboration d'une adolescente souffrant d'hystérie. L'évocation du réseau social intervient durant sa prise en charge à propos de sa relation à sa meilleure amie. Cette relation, elle l'évoque à toutes ses séances. Elle souffre de la surenchère identificatoire qu'elles nouent dans la mise à jour permanente de leur page Facebook respective. Elles se répondent (du moins est-ce le fantasme de la patiente) et se suivent quotidiennement - leur page présente le feuilleton de leur vie. Cette jeune patiente énumère combien elle ressent comme tyranniques les « retours » sur ces publications, le nombre de «like» par rapport à ceux acquis par son amie, son double. Publications de photos, mise en scène d'un quotidien supposé idéal, aux yeux des autres. Ce miroir va trouver en se brisant l'occasion d'une conflictualisation au sein de la thérapie, mais surtout d'une élaboration de sa différence et de son intériorité. C'est par un conflit à propos d'un $\mathrm{MP}^{18}$ que ses associations se libéreront et qu'il deviendra possible d'interroger en s'en différenciant, l'image construite sur ce réseau social dans le miroir imaginaire d'un autre, tyrannique dans son exigence de conformité standardisée. La critique dont à fait l'objet ma patiente se trouvait porter sur ses origines. Son amie s'était permise de pointer un trait de son caractère auprès d'une connaissance commune, en assimilant son attitude à son pays d'origine. Cette différence pointée touche au narcissisme des petites différences,

18. MP : message privé. 
mais à l'occasion de ce récit, il est intéressant de noter que si la blessure atteint ce narcissisme, (il en provient tout autant) c'est aussi à travers les ressources de ce même mécanisme que le dépassement de la souffrance va témoigner d'un processus de subjectivation, à travers l'élaboration de l'effet spéculaire et de l'agressivité qu'elle a pu révéler à cette occasion. de ce conflit, rien ne transparaîtra sur la toile. Seul fait notable: le changement de position de ma patiente par rapport à son compte Facebook, qu'elle consulte désormais de façon quantitativement moins inquiétante, sans se soucier de son taux de viralité, ou de la mise à jour de sa page.

L'aspect imaginaire que revêt la popularité des comptes Facebook m'est apparu dans le récit d'un autre adolescent au profil tout à fait différent. Pour ce patient, au profil obsessionnel, Facebook est d'autant plus important qu'il lui est impossible de se créer un compte. dans son esprit, c'est une expérience de socialisation essentielle, l'indice d'une popularité que mesure le nombre d'amis qu'arborent certaines de ces pages appartenant à ses camarades de lycée. L'évocation du réseau social revient souvent dans les propos de ce patient, obnubilé par ses difficultés relationnelles. Très inhibé, il préfère les réseaux sociaux anonymes où le mode d'interaction se fait à partir d'un pseudonyme. Il anticipe, fantasme ce qu'il ressentirait s'il était retrouvé par des copains de classe l'ayant mal traité durant la période de collège. Pire, que se passerait-il s'il avait peu d'amis ou si certains lui refusaient sa demande? Mieux vaut selon lui renoncer à se créer un compte. dans une étude de Bevan, J. L., Pfyl, J., \& Barclay, B. (n.d.), «Negative emotional and cognitive responses to being unfriended on Facebook: An exploratory stud ${ }^{\mathbf{1 9}}$, les auteurs relèvent des effets liés au rejet au sein de Facebook caractérisé par le fait d'être refusé comme ami, suite à une demande. Ces expériences sociales sont pour les adolescents plus que pourd'autres particulièrement difficiles à vivre, tant la blessure narcissique ressentie à l'occasion brise le miroir positif qu'ils viennent rechercher dans ces réseaux.

Ces deux récits caractéristiques montrent que l'enjeu narcissique propre aux réseaux sociaux n'est pas dénué de conflits. Cette conflictualité touche à la différence et se joue dans l'isolement du sujet face à l'écran. La pacification apparente du réseau s'appuie sur cette violence spéculaire où l'agressivité, notamment à l'heure du pubertaire, s'exprime par l'indifférence et la promotion de soi au détriment du souci de l'autre. L'autre, dans la page Facebook, peut bien souvent n'être que le reflet d'un souci de viralité. Le meilleur comme le pire se côtoie dans le «meilleur des mondes »: il est vrai que c'est par ce même mécanisme de propagation des messages que la révolution dans le monde arabe a pu se faire. Identification de masses, utile contre les dictatures ou pour partager

19. Bevan, J. L., Pfyl, J., \& Barclay, B. (n.d.), Negative emotional and cognitive responses to being unfriended on Facebook: An exploratory study. Computers in Human Behavior, 28(4), 1458-1464. 
des idées, mais potentiellement indigente et pauvre au cœur de nos démocraties contemporaines. Le principe économique (au sens freudien) de ces usages peut être le signe de démarcation qui permet d'y repérer des tendances pathologiques.

\section{ACTUALITÉ dU NARCISSISME dES PETITES dIFFÉRENCES}

Les processus et les mécanismes qui sont mobilisés dans le travail de culture, notamment avec la place acquise aujourd'hui par le virtuel, sont à repérer dans les nouvelles pratiques, les nouveaux usages et codes relationnels. Notre société ouverte et mondialisée offre aux amis à travers le monde de rester en lien, de se donner des nouvelles. L'abolition de la distance à travers ce canal constitue sans doute l'un des progrès les plus intéressants offerts par Facebook. Pour autant, les réseaux sociaux témoignent d'expériences dont l'analyse mérite d'être tentée.

Freud, à partir de Pour introduire le narcissisme ${ }^{\mathbf{2 0}}$, propose que le conflit psychique ne se comprenne plus comme opposition entre les nécessités de l'autoconservation et des pulsions sexuelles. deux courants libidinaux se dessinent, l'un consacré au Moi et l'autre orienté vers les autres. Ce qui est fondamental c'est que le conflit se trouve désormais pensé entre investissements narcissiques et objectaux. Il convient aujourd'hui de repenser les modalités relationnelles à partir des investissements en jeu au sein des espaces virtuels. Le courant libidinal à l'œuvre au sein des réseaux sociaux est-il fondamentalement orienté vers les autres? L'utopie sociale semble pointée vers cette hypothèse: tout se passe comme si l'amour du prochain, et les identifications garantissaient l'évitement de toute manifestation haineuse et d'annulation de l'autre.

L'aconflictualité apparente des réseaux sociaux tels que Facebook est suspecte. Non pas qu'il s'agisse de se prononcer pour ou contre un fait social d'une telle ampleur, mais de lever le refoulement qu'il concrétise pour en penser la particularité. Le narcissisme des petites différences est le plus souvent convoqué comme concept pour penser les conflits identitaires. L'omnipotence du narcissisme primaire se trouve confronté aux limitations de la réalité et à la résistance des autres à s'ajuster exactement à ses désirs et ses besoins. Or, cette résistance est largement édulcorée par la construction sociale rendue possible par Facebook où l'altérité se construit - se paramètre - au profit d'une expérimentation la plus flatteuse possible - «please like my page !». L'équilibre entre les investissements narcissiques et objectaux comporte ainsi l'investissement d'une forme d'identité par rapport à autrui qui s'exprime par le souci d'être diffèrent des autres, de ne pas être assimilé à autrui, et donc par une forme de souci du dissemblable. Cette dissemblance se construit sur fond de similitude d'une

20. Freud, Pour introduire le narcissisme, Euvres complètes Psychanalyse : Volume 12, 1913-1914. PUF. 
page à l'autre du réseau social. C'est là que le narcissisme des petites différences prend toute son ampleur. Au plan collectif, le narcissisme des petites différences organise des mépris réciproques entre des groupes sociaux qui ne sont pas si différents que cela les uns des autres. Pourtant tout est fait dans ces réseaux sociaux pour éviter, gommer, supprimer tout ce qui peut heurter cette quiétude narcissique.

Le regard de l'autre - des autres - mobilise la mise en scène des profils des comptes Facebook. La ressemblance, elle, se fonde sur le graphisme unique et sobre de la page. Jamais séparé des petits autres qui ont jalonné l'histoire identificatoire du sujet, l'usager du compte Facebook peut se tenir chaudement à l'abri de la haine, des attaques voire tout simplement de toute expérience désagréable liée à la différence. dans un article fameux G.O Gabbard ${ }^{21}$ met en avant le désappointement ressenti lorsque, malgré le souhait de similitude, le sujet trouve des différences dans l'objet aimé. Le processus de séparation et d'autonomie dans la vie du sujet, tant à la période œdipienne que préœdipienne, témoigne de cette expérience de désarroi, signe du narcissisme des petites différences selon l'auteur. Cette idée se retrouve dans l'enquête menée par les auteurs chinois Ong, Ang, Ho, Lim, Goh, Lee\& Chua ${ }^{22}$, à travers la quête de la mise en scène de « petites différences » ${ }^{23}$ par les adolescents qui se consacrent à la conception d'une page Facebook, pleinement dédiée à l'expression de soi.

dans une étude récente, "Exploring the relationship between two forms of narcissism and competitiveness $\gg{ }^{24}$, Luchner, A. F., Houston, J. M., Walker, C., \& Alex Houston, M. constatent - à partir d'une méthodologie très éloignée de la nôtre-les liens théoriques et empiriques entre le narcissisme et la compétitivité en se concentrant sur des différences individuelles caractéristiques. Basé sur un échantillon de 324 étudiants en Licence, ils mettent en évidence des relations entre le narcissisme et la compétitivité. Les découvertes mettent en relief des similarités et des distinctions entre les différentes formes de narcissisme (selon

21. Gabbard, G.O., (1993), On Hate in Love Relationships : The Narcissism of Minor differences Revisited. Psychoanal. Q., 62 :229-238.

22. Ong, E. Y.L., Ang, R. P., Ho, J. C. M., Lim, J. C. Y., Goh, d. H., Lee, C. S., \& Chua, A. Y. K. (2011). Narcissism, extraversion and adolescents' self-presentation on Facebook. Personality and Individual differences, 50(2), 180-185.

23. Ce concept n'est pas utilisé par les auteurs qui parlent de différences individuelles.

24. Luchner, A. F., Houston, J. M., Walker, C., \& Alex Houston, M. (2011). Exploring the relationship between two forms of narcissism and competitiveness. Personality and Individual differences, 51(6), 779-782. L'étude présente des mesures réduites de narcissisme manifeste (Stock (Inventaire) de Personnalité Narcissique, NPI ; Raskin et Terry, 1988) et narcissisme caché (Échelle de Narcissisme Hypersensible, HSNS ; Hendin et Joue, 1997) avec des mesures de compétitivité (Index (Indice) de Compétitivité Révisé, CI-R; Houston, Harris, McIntire et Francis, 2002) et hyper-compétitivité (Échelle d'Attitude Hypercompétitive, HCS ; Ryckman, Marteau, Kaczor et Or, 1990). 
des échelles de mesure) et la compétitivité. Ce qui est intéressant dans cette étude, c'est qu'elle témoigne, à partir d'enquêtes, de la conflictualité qui préside au sein de notre civilisation contemporaine et qui comme on le voit, s'articule avec le narcissisme, compris il est vrai dans un sens bien différent de la psychanalyse. Quoiqu'il en soit, ils épinglent en quelque sorte le malaise propre à notre époque. Or, dans Malaise dans la civilisation, Freud avance l'idée selon laquelle le narcissisme des petites différences témoigne de l'impossibilité d'aimer son prochain comme soi-même. de cette impossibilité, Facebook offre un intermédiaire palliatif, une zone d'apparente aconflictualité, au cœur de laquelle, soimême, comme chaque autre, peut voir sans être vu ou être vu sans jamais rien voir. Tel est le paradoxe qu'offrent les réseaux sociaux comme asile à toute ambivalence.

Rémy POTIER

42, Bd de Port-Royal

75005 Paris

remy.potier@univ-paris-diderot.fr

\section{BiBliograPhie}

Antoci, A., Sabatini, F., \& Sodini, M., (2012), See you on Facebook! A framework for analyzing the role of computer-mediated interaction in the evolution of social capital. The Journal of Socio-Economics, 41(5), 541-547.

Batout, J., (2011), Le monde selon Facebook, Le Débat, 163.

Bevan, J. L., Pfyl, J., \& Barclay, B., (n.d.), Negative emotional and cognitive responses to being unfriended on Facebook: An exploratory study. Computers in Human Behavior, 28(4), 1458-1464.

FreUd, Pour introduire le narcissisme, Euvres complètes Psychanalyse: Volume 12, 19131914, PUF.

Freud, S., (1918), « Le tabou de la virginité » traduit de l'allemand par Janine Altounian, André Bourguignon, Pierre Cotet... [et al.]; préface de Christophe Jouanlanne, in Contributions à la psychologie de la vie amoureuse, Paris, PUF, 2011.

Freud, S., (1921/1981) Psychologie des foules et analyse du moi, - trad. P. Cotet, A. et O. Bourguignon, J. Altounian, A. Rauzy, in Essais de psychanalyse, Paris, Payot, 1981.

FreUd, S., (1929), Malaise dans la civilisation, trad. Ch. et J. Odier, Paris, PUF, France, 1979.

Gabbard, G.O., (1993), On Hate in Love Relationships : The Narcissism of Minor differences Revisited. Psychoanal. Q., 62 :229-238.

GonZALES AL, Hancock JT. Mirror, mirror on my Facebook wall : effects dexposure to Facebook on self-esteem. Cyberpsychol Behav Soc Netw $2011 ; 14: 1-2$. 
GutTon, P., Le pubertaire, Le pubertaire. (2003), PUF.

LACAN, J., L'agressivité en psychanalyse, Écrits, Seuil.

Leroi Ghouran, A., (1964-1965), Le Geste et la Parole, 1. : Technique et langage, 2. : La Mémoire et les Rythmes, Paris, Albin Michel,

Luchner, A. F., Houston, J. M., Walker, C., \& Alex Houston, M. (2011). Exploring the relationship between two forms of narcissism and competitiveness. Personality and Individual differences, 51(6), 779-782.

Missonnier, S., et Lisandre, H., (sous la direction de), (2003), Le virtuel - la présence de l'absent, Paris, EdK.

Moreau, A., Roustit, O., Chauchard, E., \& Chabrol, H. (2012). L'usage de Facebook et les enjeux de l'adolescence : une étude qualitative. Neuropsychiatrie de l'enfance et de l'adolescence, 60 (6), 429-434.

Ong, E. Y. L., Ang, R. P., Ho, J. C. M., Lim, J. C. Y., Goh, d. H., Lee, C. S., \& ChuA, A. Y. K. (2011). Narcissism, extraversion and adolescents self-presentation on Facebook. Personality and Individual differences, 50(2), 180-185.

Piotet, d., (2011), Comment les réseaux sociaux changent notre vie. Esprit, Juillet (7), p. 82.

Potier, R., \& Bialès, P., (2007), de l'amitié en virtuel. Adolescence, 61(3), 581.

Potier, R., (2009), Au risque du virtuel, Topique 2/2009 ( $\mathrm{n}^{\circ}$ 107), p. 149-162.

Potier, R., (2009), L’image en médecine, esquisse et précipice, Recherches en Psychanalyse, 8

PotIER, R., (2011), Imagerie médicale et art contemporain, rencontres autour d'un corps virtuel. Recherches en Psychanalyse, 12.

RichaRd, F., (2011), Les formes actuelles du malaise dans la culture. Recherches en Psychanalyse, 11.

rémy PoTier - Facebook à l'épreuve de la différence. Avatars du narcissisme des petites différences

résumé : Les réseaux sociaux, tels que Facebook sont de plus en plus investis par les usagers d'internet. Ces usages témoignent du meilleur comme du pire, mais demandent surtout à être élucidés du point de vue de la psychanalyse. Le concept de « narcissisme des petites différences » est de ce point de vue tout à fait pertinent. Il permet de saisir l'enjeu narcissique de l'aconflictualité de surface dont témoigne en apparence cet espace d'échange. Il s'agira de mettre à l'épreuve ce concept à partir de la nouveauté que caractérisent les modalités relationnelles liées au virtuel. La psychanalyse peut ainsi se saisir d'études pluridisciplinaires traitant de Facebook pour l'articuler à partir de la clinique aux enjeux psychiques qui ne manquent pas de ce faire jour dans ce contexte.

Mots-clés : Facebook - Virtuel - Narcissisme des petites différences - Identification - Agressivité - Interdisciplinarité. 


\section{rémy PoTier-Facebook Put to the Test of Difference. Avatars of the Narcissism of SmallDifferences.}

abstract : Social networks like Facebook have an ever-increasing number of adepts and, while they are used in ways which bear witness to both the worst and the best in humankind, they also demand a degree of analysis from the psychoanalytical point of view. The concept of the 'narcissism of small differences' is of great use in this light, enabling us to seize upon the narcissistic stakes at play in the apparently a-conflictual world these interfaces of exchange seemingly establish. This article tests this concept against the yardstick of the novelty inherent in the relational modalities of the virtual realm. Psychoanalysis draws upon pluri-disciplinary studies on Facebook to examine this phenomenon from the standpoint of the clinical practice of the psychical processes this specific context brings to light.

Key-words : Facebook - Virtual - Narcissism of Small differences - Identification Aggressiveness - Inter-disciplinarity. 